\title{
Analysis of the Quantum Spin Hall and Quantum Anomalous Hall Effects in a Two-Dimensional Decorated Lattice Using Entanglement Spectrum
}

\author{
A. Piekarska, P. Potasz and A. Wójs \\ Department of Theorethical Physics, Wrocław University of Technology, \\ Wybrzeże Wyspiańskiego 27, 50-370 Wrocław, Poland
}

\begin{abstract}
We study topological properties of a two-dimensional decorated lattice using entanglement spectrum and trace index. Using these measures, we distinguish various phases of the system predicted recently, including the quantum spin Hall effect and quantum anomalous Hall effect. The topologically trivial and non-trivial phases are identified, with assignment of a Chern number to each of the bands.
\end{abstract}

DOI: 10.12693/APhysPolA.129.A-87

PACS: 73.20.At, 73.43.Cd

\section{Introduction}

Since the discovery of the quantum Hall effect (QHE) in 1980 [1], topological states of matter have been extensively investigated $[2,3]$. A full family of quantum Hall like states was recently discovered experimentally, including quantum spin Hall effect (QSHE) and quantum anomalous Hall effect (QAHE) [4-6].

QHE-like states are examples of topologically nontrivial phases, distinguished from trivial ones by a topological invariant. They are characterized by insulating bulk and edge states that are responsible for the transport along the edges.

In most cases the knowledge of all expectation values of operators in the ground state is enough to classify the state of matter. However, it is not true of the "topological phases", for which more sophisticated measures are needed to distinguish between distinct topological classes. Topological properties of a system can be found using entanglement measures [7]. One of the advantages of entanglement is that it can reveal non-local correlations in the system, which sometimes cannot be obtained from its band structure.

In this work, by using entanglement spectra, we analyze the existence of QSHE and QAHE for a lattice model with non-trivial topology of energy bands. We consider decorated square lattice (called the Lieb lattice) [8]. Following Zhao and Shen [9], the Chern number of the energy bands depends on the relative strength of the complex hopping constant [10] and the staggered potential amplitude. We analyze the energy and entanglement spectra of the system in strip geometry for various strengths of the staggered potential. We identify the topologically trivial and non-trivial phases, obtaining the regimes of parameters for which QSHE and QAHE states are possible.

\section{Methodology}

\subsection{Model}

The decorated lattice is a two-dimensional square lattice, with an additional atom between each pair of nearest neighbors, as shown in Fig. 1a. The lattice can be divided into two sublattices $\mathbb{A}$ and $\mathbb{B}$, distinguished by filled and empty circles. In the tight-binding approach we include the nearest neighbor hopping term, with amplitude $t$, and the spin-orbit coupling term, with amplitude $\lambda$,

$$
H=t \sum_{\langle i j\rangle \sigma} c_{i \sigma}^{\dagger} c_{j \sigma}+i \lambda \sum_{\langle\langle i j\rangle\rangle \alpha \beta} v_{i j} c_{i \alpha}^{\dagger} \sigma_{\alpha \beta}^{z} c_{j \beta},
$$

where $v_{i j}= \pm 1$ depending on the chirality of the jump, $\sigma^{z}$ is the Pauli matrix and $c_{i \alpha}$ is the annihilation operator for a particle with spin $\alpha$ at the site $i$.

The unit cell consists of three sites giving three energy bands. In the nearest neighbor approximation, when only the first term of Eq. (1) is considered, the band structure consists of two dispersive bands touching each other at energy $E=0$, where a totally flat third energy band is present $[8,11]$. Inclusion of the second term opens the energy gap between the two dispersive bands, additionally making them topologically non-trivial with the Chern numbers $C= \pm 1(C=\mp 1)$ for spin-up (spindown) bands, depending on the choice of the phase sign. A nondispersive zero-energy band remains topologically trivial with the Chern number $C=0$.

To allow manipulation of the Chern numbers of the bands, the staggered potential terms are introduced to the Hamiltonian. As was shown by Zhao and Shen [9], variation of the strength of the potential closes the energy gap and re-opens it, swapping the Chern numbers of the energy bands at their crossing point. The staggered potential depends on the sublattice $(\mathbb{A}$ or $\mathbb{B})$ and consists of the spin-dependent part

$$
H_{s}=-v_{s} \sum_{i \in \mathbb{A}} c_{i}^{\dagger} \sigma^{z} c_{i}+v_{s} \sum_{i \in \mathbb{B}} c_{i}^{\dagger} \sigma^{z} c_{i}
$$


and the spin-independent part

$$
H_{c}=-v_{c} \sum_{i \in \mathbb{A}} c_{i}^{\dagger} c_{i}+v_{c} \sum_{i \in \mathbb{B}} c_{i}^{\dagger} c_{i},
$$

where $v_{s}$ and $v_{c}$ are strengths of spin-dependent and spinindependent parts, respectively. We may introduce variables $S_{ \pm}$such that

$$
S_{ \pm}=v_{s} \pm v_{c} .
$$

These parameters are more convenient since $S_{+}\left(S_{-}\right)$only enters the spin up (down) part of the Hamiltonian and therefore we can treat them separately.

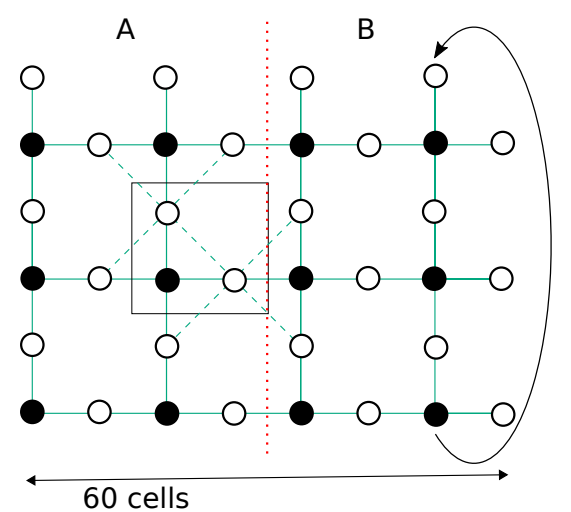

Fig. 1. The investigated system. The square box contains the unit cell. The system is a strip 60 cells wide and infinitely long, modeled by periodic boundary conditions. The red dotted line shows how the system is divided into parts A and B. The solid color lines denote the nearest neighbor hopping and the dashed color lines denote spin-orbit interaction. The sublattices $\mathbb{A}$ and $\mathbb{B}$ are marked by black (filled) and white (empty) dots, respectively.

In our calculations, we are dealing with an infinite strip of 60 unit cells width. The regions A and B are the left and right sides of the strip and their widths are equal, as pictured in Fig. 1a. In this system, $k_{y}$ is a good quantum number and we Fourier transform the Hamiltonian in the $y$ direction.

\subsection{Entanglement spectrum}

We consider a system in the many-body ground state $\left|\Psi_{\mathrm{GS}}\right\rangle$, which is given by a Slater determinant of the single-particle states of all the states below the Fermi level. The density matrix of the whole system in this state is $\rho=\left|\Psi_{\mathrm{GS}}\right\rangle\left\langle\Psi_{\mathrm{GS}}\right|$. Each density matrix corresponds to some Hamiltonian, via the thermodynamical relation

$$
\rho=\mathcal{N} \exp (-\beta H),
$$

where $\beta=\frac{1}{k_{\mathrm{B}} T}$ is the Boltzmann factor and $\mathcal{N}$ is a normalization factor. The system can be divided into two parts, A and B, as shown in Fig. 1a. We can define the reduced density matrix of the subregion $\mathrm{A}$ as $\rho_{\mathrm{A}}=\operatorname{Tr}_{\mathrm{B}} \rho$. We are interested in the entanglement between those two parts. We define the entanglement Hamiltonian as the Hamiltonian corresponding to $\rho_{\mathrm{A}}$ in unit temperature.
The set of eigenvalues of this Hamiltonian is called the entanglement energies and denoted by $\left\{\xi_{i}\right\}$.

The entanglement energies are related to the eigenvalues $\left\{\zeta_{i}\right\}$ of the reduced correlation matrix [12]:

$$
C_{i j}=\left\langle\Psi_{\mathrm{GS}}\left|c_{i}^{\dagger} c_{j}\right| \Psi_{\mathrm{GS}}\right\rangle, \quad i, j \in[1, N / 2]
$$

by the formula

$$
\zeta_{i}=\frac{1}{e^{\xi_{i}}+1} .
$$

These eigenvalues are called the single particle entanglement spectrum. A system in a topologically non-trivial phase cannot be continuously transformed to a system with zero entanglement without closing the energy gap. This situation holds if and only if the entanglement spectrum is gapless.

Another measure that can be obtained from the singleparticle entanglement spectrum is the trace index [7] defined as:

$$
\operatorname{Tr}(C(k))=\sum_{i=1}^{N / 2}\left\langle\Psi_{\mathrm{GS}}\left|c_{i}(k)^{\dagger} c_{i}(k)\right| \Psi_{\mathrm{GS}}\right\rangle .
$$

If this measure has a discontinuity for some $k$ then the Chern number is equal to the difference of the right and left limits at this point. Otherwise, the Chern number $C=0$.

\section{Results}

In this section, we show that various quantum phases can be distinguished. In Fig. $2 \mathrm{~b}$ we deal with spin up electrons for $S_{+}>2 \lambda$. The fillings are $\nu=1 / 3$ (top row) and $\nu=2 / 3$ (bottom row) corresponding to fully filled lower band and fully filled two lower bands, respectively. The energy band structure is shown in the first column from the left. A characteristic feature is a presence of edge states between the middle and the upper band. In the second column the single-particle entanglement spectrum $\left\{\zeta_{i}\right\}$ is shown. For the filling factor $\nu=1 / 3$ (top row), the spectrum is gapped which means that the lower band is topologically trivial. For the filling factor $\nu=2 / 3$ (bottom row), the spectrum is gapless which means that the lower and middle bands together are topologically non-trivial [7]. The third column shows the entanglement energies $\left\{\xi_{i}\right\}$ which are related to the entanglement spectrum by the formula $\xi_{i}=\ln \left(1-1 / \zeta_{i}\right)$. The third column contains the same information as the second one but allows one to have closer insight into the inner structure of the entanglement energy bands. The fourth column pictures the trace index. For the filling factor $\nu=1 / 3$ there are no discontinuities which means the Chern number of the lower band is zero. For the filling factor $\nu=2 / 3$ there is a discontinuity. The right limit at this point is equal 59.5 and the left is 60.5, therefore the Chern number for the lower and middle bands together is $C=59.5-60.5=-1$. Since the Chern number of the bottom band was $C=0$, we know that the middle band Chern number has to be equal $C=-1$. Since the Chern number of the whole system must be 0 , the Chern number of the upper band is $C=1$. 

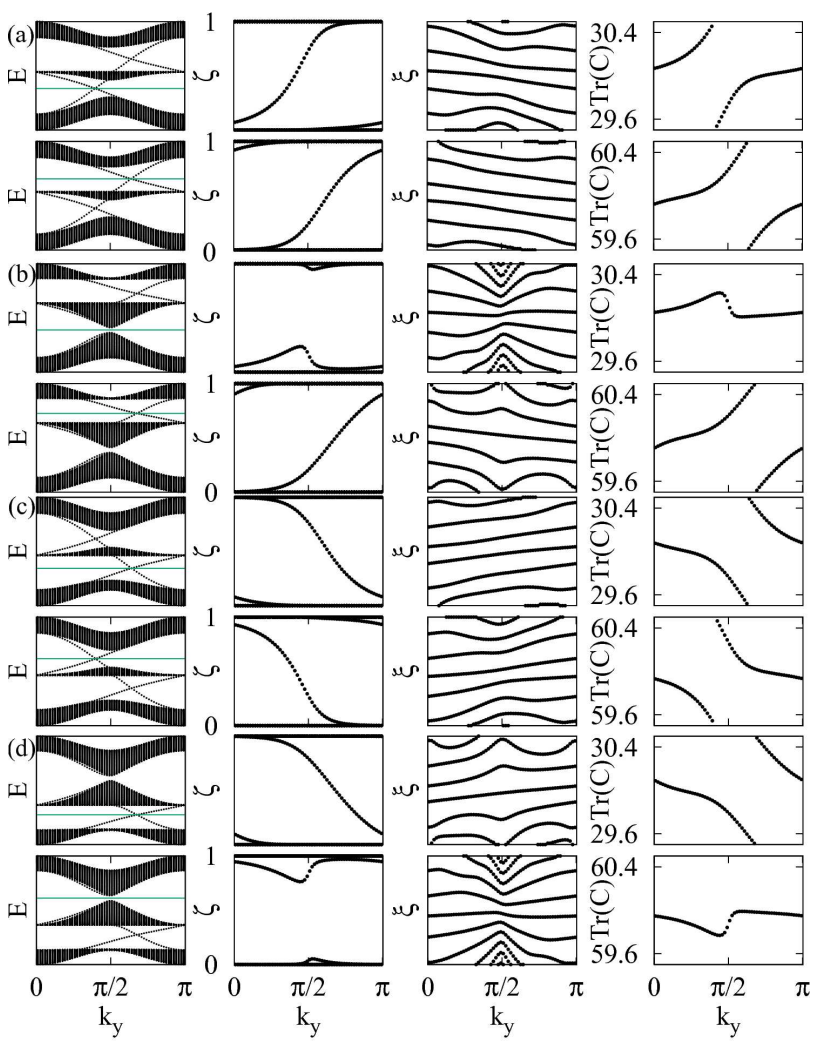

Fig. 2. Results for (a,b) spin up and (c,d) spin down with (a,c) $S_{ \pm}<2 \lambda$ and (b,d) $S_{ \pm}>2 \lambda$ for filling $1 / 3$ (top row) and $2 / 3$ (bottom row). The first column from the left shows the energy spectrum, with the horizontal green line indicating the Fermi level. The second and third columns from the left present the entanglement spectrum and entanglement energies. The fourth column shows the trace index. The Chern numbers are (a) -1 for both fillings, (b) 0 and -1 for fillings $1 / 3$ and $2 / 3$, respectively, (c) 1 for both fillings and (d) 1 and 0 for fillings $1 / 3$ and $2 / 3$, respectively.

Similar analysis of Fig. 2a-d provides the results gathered in Table I. From these results we can see that changing from $S_{+}\left(S_{-}\right)<2 \lambda$ to $S_{+}\left(S_{-}\right)>2 \lambda$ for spin up (down) changes the Chern numbers of the bands. Therefore we can manipulate the Chern numbers of chosen bands using various strengths of the staggered potential. Note that since $S_{ \pm}=v_{s} \pm v_{c}$, we can select both $S_{+}$ and $S_{-}$independently.

\section{TABLE I}

The Chern numbers for the three bands depending on the choice of parameters. The symbols $S_{ \pm}$and $\lambda$ are defined in the text.

\begin{tabular}{c|c|c|c|c}
\hline \hline \multicolumn{2}{c|}{ Parameters } & \multicolumn{3}{c}{ Chern number for band } \\
\hline Spin & Potential & Lower & Middle & Upper \\
\hline up & $S_{+}<2 \lambda$ & -1 & 0 & 1 \\
up & $S_{+}>2 \lambda$ & 0 & -1 & 1 \\
down & $S_{-}<2 \lambda$ & 1 & 0 & -1 \\
down & $S_{-}>2 \lambda$ & 1 & -1 & 0
\end{tabular}

Based on this analysis, it is possible to obtain various types of the quantum phases. For example choosing $S_{+}<2 \lambda, S_{-}<2 \lambda$ and filling factor $\nu=1 / 2$ will result in total Chern number $C=0$. However the Chern numbers for single spins will be $C=+1$ and $C=-1$ so the system will exhibit QSHE. On the other hand, if we choose $S_{+}>2 \lambda, S_{-}<2 \lambda$ and filling factor $\nu=1 / 2$ and shift the energies for the two spins such that the middle band for the spin down is below the middle band for the spin up, we will obtain the total Chern number $C=1$ and therefore the system will exhibit QAHE, confirming results obtained by Zhao and Shen [9].

\section{Conclusions}

We have presented entanglement spectrum and trace index for various cases of spin, filling and parameter choice for the decorated lattice with staggered potential. This allowed us to identify the topologically trivial and non-trivial phases and assign a Chern number to each of the bands. Our analysis confirms predictions made by Zhao and Shen [9] showing that depending on parameters values the system may exhibit QSHE or QAHE.

\section{Acknowledgments}

The authors acknowledges partial financial support from National Science Center (NCN), Poland, grant Sonata No. 2013/11/D/ST3/02703.

\section{References}

[1] K. von Klitzing, G. Dorda, M. Pepper, Phys. Rev. Lett. 45, 494 (1980).

[2] C.L. Kane, E.J. Mele, Phys. Rev. Lett. 95, 146802 (2005).

[3] M.Z. Hasan, C.L. Kane, Rev. Mod. Phys. 82, 3045 (2010).

[4] M. König, S. Wiedmann, C. Brüne, A. Roth, H. Buhmann, L.W. Molenkamp, X.L. Qi, S.C. Zhang, Science 318, 766 (2007).

[5] S. Oh, Science 340, 153 (2013).

[6] C.-Z. Chang, J.S. Zhang, X. Feng, J. Shen, Z.C. Zhang, M.H. Guo, K. Li, Y.B. Ou, P. Wei, L.L. Wang, Z.Q. Ji, Y. Feng, S.H. Ji, X. Chen, J.F. Jia, X. Dai, Z. Fang, S.C. Zhang, K. He, Y.Y. Wang, L. Lu, X.C. Ma, Q.K. Xue, Science $\mathbf{3 4 0}$ 167 (2013).

[7] A. Alexandradinata, T.L. Hughes, B.A. Bernevig, Phys. Rev. B 84, 195103 (2011).

[8] C. Weeks, M. Franz, Phys. Rev. B 82, 085310 (2010).

[9] A. Zhao, S.Q. Shen, Phys. Rev. B 85, 085209 (2012).

[10] F.D.M. Haldane, Phys. Rev. Lett. 61, 2015 (1988).

[11] M. Niţă, B. Ostahie, A. Aldea, Phys. Rev. B 87, 125428 (2013).

[12] I. Peschel, J. Phys. A Math. Gen. 36, L205 (2003). 